\title{
Conference Paper Noncanonical Bases of Cycle and Cutset Spaces of Graphs
}

\author{
J. M. S. Simões-Pereira \\ Departamento de Matemática, Universidade de Coimbra, Portugal \\ Correspondence should be addressed to J. M. S. Simões-Pereira; siper@mat.uc.pt
}

Received 12 March 2013; Accepted 22 May 2013

Academic Editors: B. Datta, G. S. F. Frederico, N. Martins, D. F. M. Torres, and A. J. Zaslavski

This Conference Paper is based on a presentation given by J. M. S. Simões-Pereira at "The Cape Verde International Days on Mathematics 2013" held from 22 April 2013 to 25 April 2013 in Praia, Cape Verde.

Copyright (C) 2013 J. M. S. Simões-Pereira. This is an open access article distributed under the Creative Commons Attribution License, which permits unrestricted use, distribution, and reproduction in any medium, provided the original work is properly cited.

We characterize those bases of the cycle space and of the cutset space of a graph $G$ which cannot be associated with a spanning tree of $G$.

The so-called cycle space of a (connected) graph $G=(V, E)$ is well known (see Harary [1]). Remember the definitions: A (not necessarily connected) subgraph of $G$ will be called Eulerian if, when regarded as a graph, all its vertices are of even degree. We take the edge sets of such subgraphs (not all of them are cycles, obviously!) as the vectors of the cycle space: the vector sum is the ring sum or symmetric difference, the scalar field is $\mathscr{F}_{2}=\{0,1\}$; the product of a scalar by a vector $X$ is defined by setting $0 \otimes X=\emptyset$ and $1 \otimes X=X$. By convention, we consider the empty set as the null vector of this space.

Given a (connected) graph $G$ and one of its spanning trees $T$, we call the edges in $T$ branches and the remaining edges of $G$, chords. For each chord, if we add it to $T$, we form a cycle, and the set of the $|E(G)|-|E(T)| \equiv|E(G)|-|V(G)|+1$ cycles formed in this way may serve as a basis (or fundamental set) of the cycle space. Such bases will be said to be canonical, but there are bases which cannot be associated with any tree: we call them noncanonical.

The important role assumed by spanning trees when we look for bases of the cycle space (or of the cutset space) of a graph is unquestionable. It is revealing that, while investigating cycle spaces of infinite graphs, Vella and Richter [2] (and several other authors they quote) feel the need to generalize the concept of spanning tree in a way that makes sense in infinite graphs.

Some authors (see Ponstein [3], Seshu and Reed [4]) seem however to forget that there are noncanonical bases. Even in Thulasiraman [5], this fact is not pointed out. In Seshu and
Reed [4], it is said in error that "to have a basis we need a tree."

Let us give an example of a noncanonical basis: in the graph of Figure 1, the four triangles $K_{1}=\{a, b, c\}, K_{2}=$ $\{a, d, e\}, K_{3}=\{b, g, f\}$, and $K_{4}=\{c, h, i\}$ form a basis of the cycle space. They are linearly independent because $\oplus_{m=1}^{4} c_{m} \otimes$ $K_{m}=\emptyset$ requires $c_{2}=c_{3}=c_{4}=0$ so that $d, g, h$, respectively, do not appear in the sum; hence, we must have also $c_{1}=0$ to obtain $\emptyset$ as the sum.

Note that, in a canonical basis, an edge common to two cycles cannot be a chord: the other edges of these cycles would belong to the tree, a contradiction because they either form or properly contain another cycle. Now suppose that there exists a spanning tree $T$ of $G$ associated with the abovementioned four triangles. As just seen, the edges $a, b, c$ cannot be chords; that means they belong to $T$, a contradiction. In general we have the following.

Theorem 1. A necessary and sufficient condition for a basis of the cycle space to be canonical is that each one of its cycles contains one edge not belonging to anyone of the other cycles of the basis.

Proof. The necessity is obvious: if a basis is associated with a tree, then each one of its cycles contains one chord that belongs to no other cycle of the basis.

Now let $K_{1}, \ldots, K_{m}$ be the $m$ cycles of a basis where $m=|E(G)|-|V(G)|+1$ and, for $i=1, \ldots, m$, let $e_{i}$ be an 


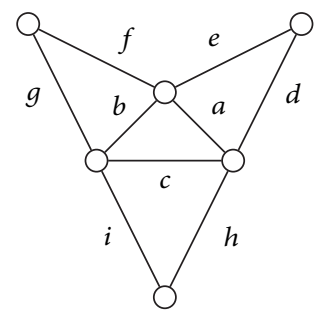

FIGURE 1

edge of $K_{i}$ that belongs to no $K_{j}$ with $j \neq i$. Let us start from $G$ and successively remove the edges $e_{i}$. We end up with a subgraph $T$ with the same number of vertices as $G$ and with $|E(G)|-m \equiv|V(G)|-1$ edges. Let us show that $T$ is connected, which implies that $T$ is a spanning tree of $G$. In fact, the removal of an edge that belongs to a cycle from a connected graph yields a new graph which is still connected; since each $e_{i}$ belongs to just one cycle $K_{i}$, the successive removals do not affect the other cycles $K_{j}(j \neq i)$ and hence no one of these removals destroys the connectivity of the graphs we successively obtain. Thus $T$ is connected that means a tree. If we add to $T$ the last $e_{i}$ we removed, say $e_{m}$, we reconstruct $K_{m}$, the last cycle destroyed by the successive removals. The point is that we may remove the edges $e_{i}$ in any order; hence, anyone of the cycles $K_{i}$ may occur as the last one. This means that, for $i=1, \ldots, m$, if we add $e_{i}$ to $T$ we obtain $K_{i}$, which proves the sufficiency of the condition.

Analogous considerations may be made for the so-called cutset (or separator) space of $G$. We distinguish cutsets and separators. In general, a separator of $G$ is a set of edges whose removal increases the number of connected components of $G$. A cutset is a separator which is minimal, that means, a separator which does not properly contain another separator.

Let $G$ be connected and $T$ a spanning tree of $G$. Let $[v, w]$ be an edge of $T$. If we remove this edge, we remain with two subtrees, one, say $T^{\prime}$, containing $v$ and the other, say $T^{\prime \prime}$, containing $w$. The edge set formed by $[v, w]$ and all chords linking one vertex of $T^{\prime}$ with one vertex of $T^{\prime \prime}$ form a cutset of $G$. These $|V(G)|-1$ cutsets may be regarded as the basis of the cutset space of $G$. The vector sum, the scalar field, and the product of a scalar by a vector are defined as for the cycle space. The empty set is also, by convention, the null vector of this space.

The vectors of the cutset space are the edge sets of $G$ that may be obtained by ring sums of basic cutsets. Obviously, some of them are separators but not cutsets. On the other hand, we point out that there are separators which do not belong to this space; they cannot be expressed as linear combinations of the basic cutsets. For example, let $G$ be a pentagon whose edges we denote by $a, b, c, d, e$ as we move around, say, clockwise. Let the edges of $T$ be $a, b, c, d$. The associated cutsets are $\{a, e\},\{b, e\},\{c, e\}$ and $\{d, e\}$. The cutsets of the pentagon are the pairs of edges. Now see that $\{a, b, c\}$ is a separator and is not a linear combination of the basic cutsets. It does not belong to the cutset space of this graph.

Here also, we distinguish canonical bases (those associated with a spanning tree) and noncanonical ones. An example of the latter is given if we choose $J_{1}=\{f, g\}, J_{2}=$ $\{h, i\}, J_{3}=\{e, d\}, J_{4}=\{f, e, a, b\}$, and $J_{5}=\{g, b, c, h\}$ for basic cutsets of the graph of Figure 1. These five sets are linearly independent: to satisfy the condition $\oplus_{m=1}^{5} c_{m} \otimes J_{m}=\emptyset$, we must choose $c_{2}=c_{3}=0$ so that neither $i$ nor $d$ appear in the sum; hence, we need also $c_{4}=c_{5}=0$ so that neither $e$ nor $h$ appears; finally, we need $c_{1}=0$ so that the sum is the empty set. The following holds.

Theorem 2. A necessary and sufficient condition for a basis of the cutset space to be canonical is that each one of its cutsets contains one edge not belonging to anyone of the other cutsets of the basis.

Proof. The necessity is obvious. When the basis is canonical, each basic cutset contains one branch of the tree which belongs to no other basic cutset.

For the sufficiency, we need the obvious fact that a cycle and a cutset of a graph share either none or an even number of edges. Now, choose in each one of the $|V|-1$ basic cutsets one edge that belongs to no other one. Let $H$ be the subgraph formed by such edges. Let us first prove that $H$ contains no cycle: if it did, no cutset could contain just one edge of such a cycle, that is, just one edge of $H$, a contradiction. Hence $H$ is a subgraph of $G$ with $|V|-1$ edges and no cycles. This means $H$ is a spanning tree of $G$. Since every edge of $H$ belongs to one of the given cutsets, we conclude that they form a canonical basis associated with the tree $H$, which proves the sufficiency.

\section{References}

[1] F. Harary, Graph Theory, Addison-Wesley, Reading, Mass, USA, 1969.

[2] A. Vella and R. B. Richter, "Cycle spaces in topological spaces," Journal of Graph Theory, vol. 59, no. 2, pp. 115-144, 2008.

[3] J. Ponstein, Matrices in Graph and Network Theory, Van Gorcum, Assen, The Netherlands, 1966.

[4] S. Seshu and M. B. Reed, Linear Graphs and Electrical Networks, Addison-Wesley, Reading, Mass, USA, 1961.

[5] K. Thulasiraman, "Graphs and vector spaces," in Handbook of Graph Theory, J. L. Gross and J. Yellen, Eds., pp. 533-556, CRC Press, New York, NY, USA, 2004. 


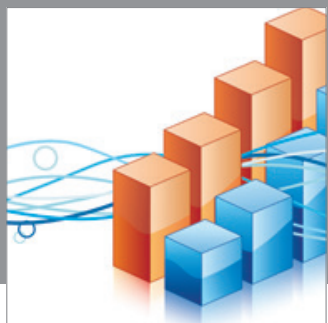

Advances in

Operations Research

mansans

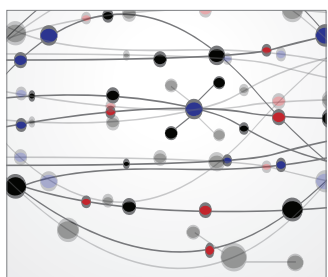

The Scientific World Journal
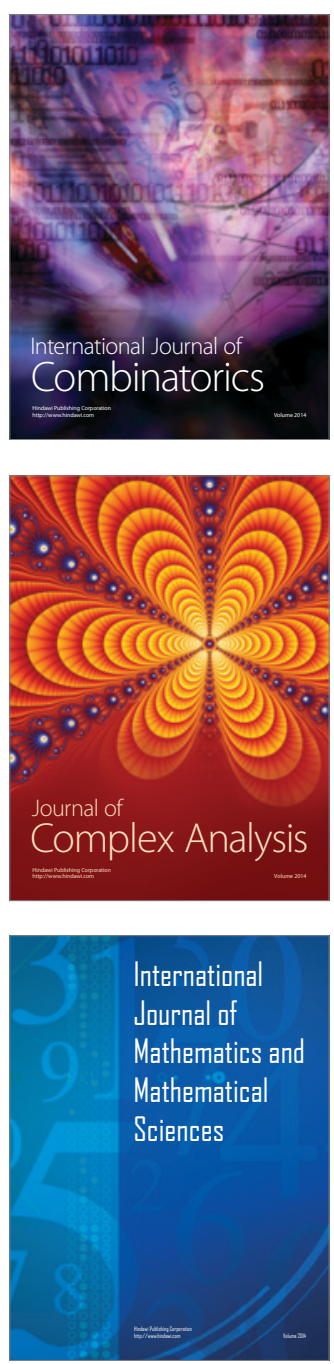
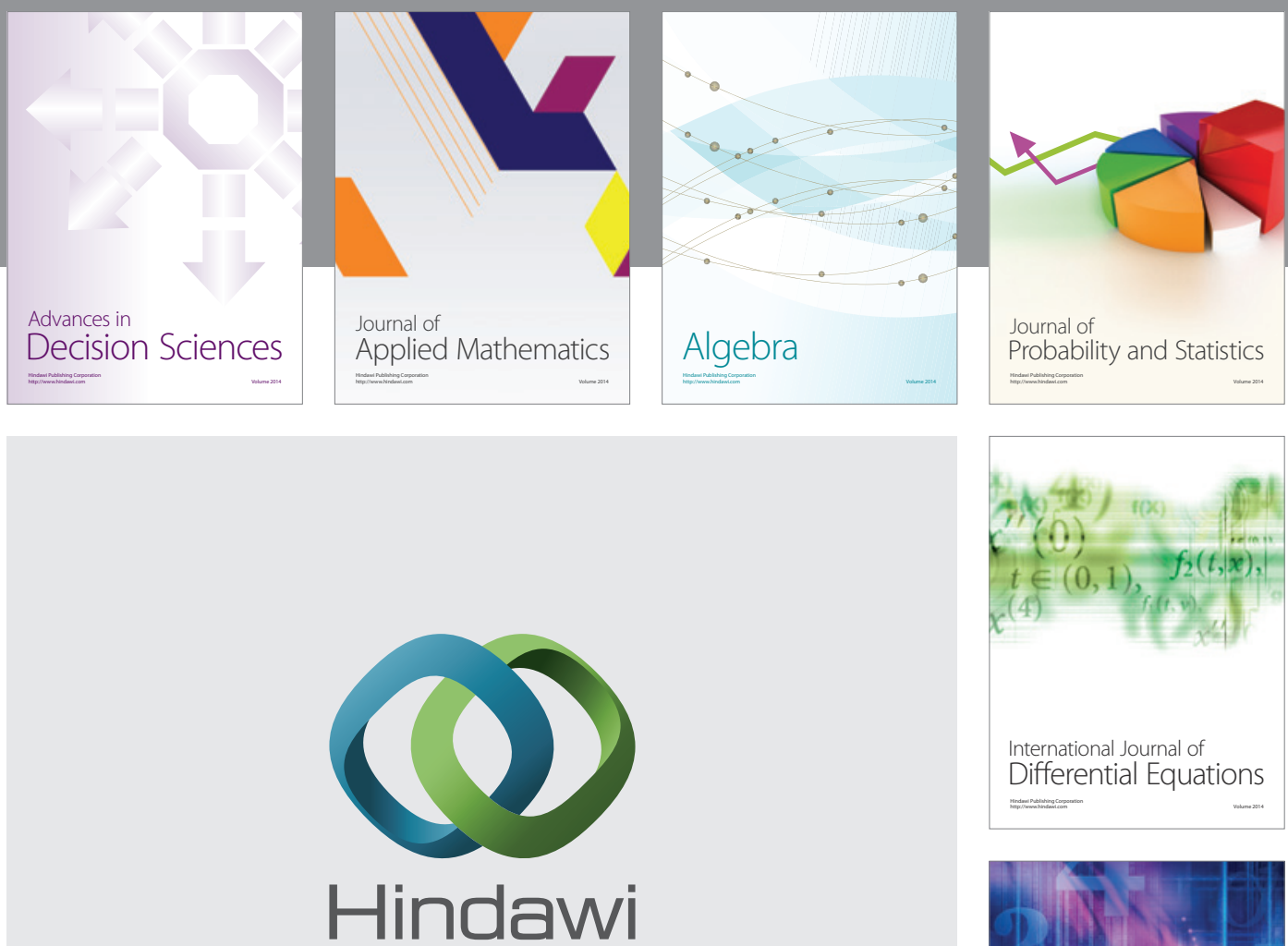

Submit your manuscripts at http://www.hindawi.com
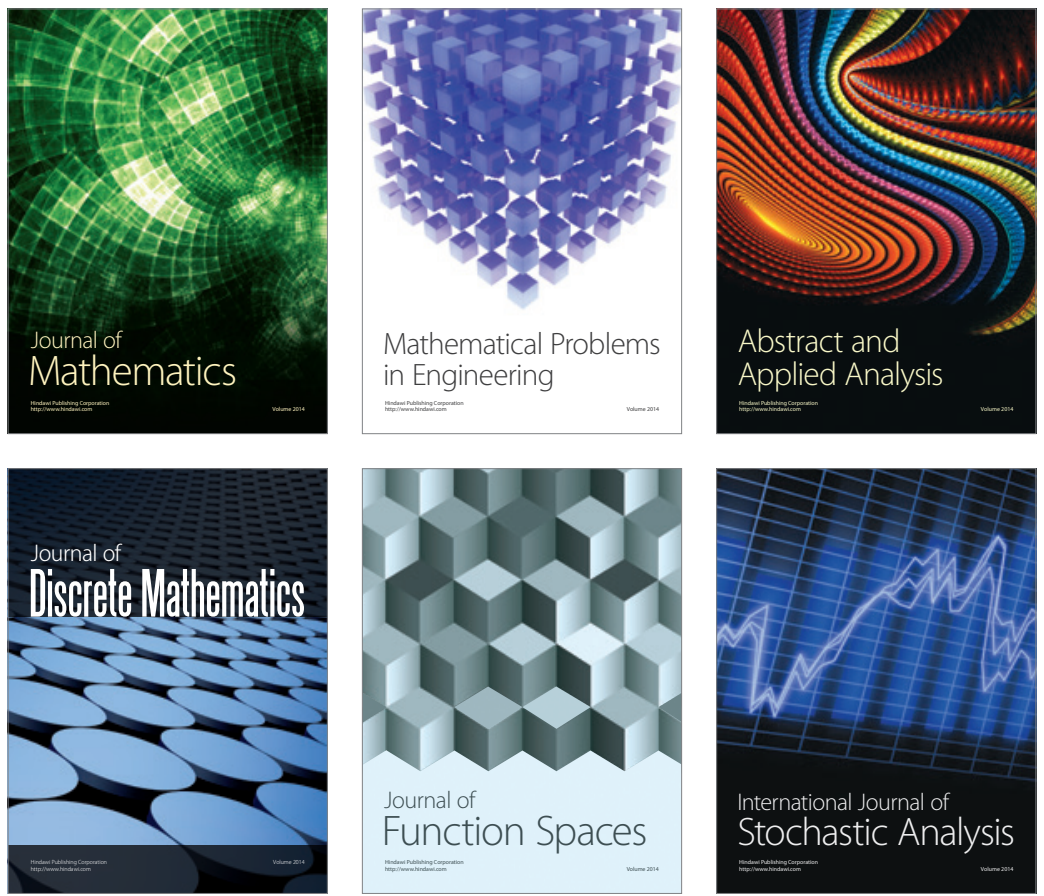

Journal of

Function Spaces

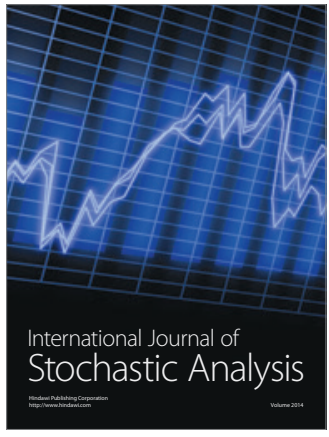

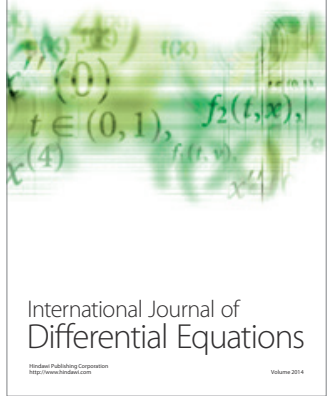
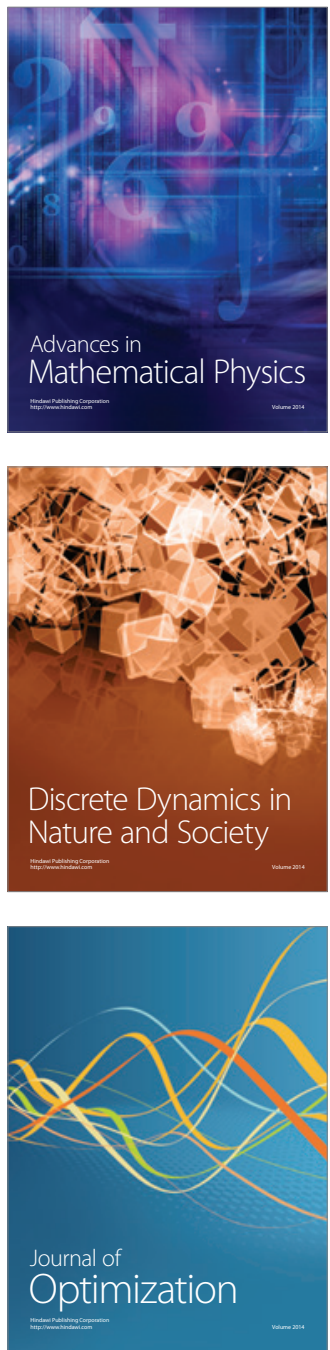